\title{
Estatus jurídicos de las personas transexuales en la Unión Europea y Latinoamérica
}

Fecha de recepción : 17 de mayo de 2019 • Fecha de aceptación: 31 de julio de 2019 • Fecha de publicación: 10 de septiembre de 2019

Dra. Janny Carrasco Medina

Universidad de Brasilia

jannycarrasco83@gmail.com

https://orcid.org/0000-0003-2183-9182

Delphine Defossez

Doctoranda de la Universidad de Brasília, Brasil

delphine.defossez@live.be

https://orcid.org/0000-0001-7285-0491

\section{Resumen}

Las personas trans están entre los miembros más vulnerables de cualquier sociedad y experimentan altos niveles de estigmatización y marginación. El presente artículo tiene como objetivo realizar un análisis de los derechos de las personas transexuales, abordando para ello tanto la jurisprudencia como la legislación en el contexto europeo y latinoamericano. Dentro del nudo cognitivo jurídico internacional y apoyado en la metodología cualitativa se realizó una caracterización de la realidad facto-jurídica de la legislación aplicable a estas personas.

\section{Palabras clave: transexualidad, jurisprudencia, identidad de género.}

\section{Abstract}

Trans people are among the most vulnerable members of any society and experience high levels of stigmatization and marginalization. This article is analyzing the rights of transsexual people, addressing both case law and legislation in the European and Latin American context. Within the international legal cognitive knot and supported by the qualitative methodology, a characterization of the legal-fact reality of the legislation applicable to these people. 


\section{Introducción}

Desde inicios del siglo XX encontramos varios estudios sobre personas transexuales, entre los que se destacan autores como Hirschfeld (1923), Cauldwell (1949), Benjamin (1966), Stoller (1982), Money (1997) y Fisk (1973), los cuales crean el concepto de disforia de género. Todos ellos de cierta forma contribuyeron a que, en 1987, la transexualidad fuera incluida en el Diagnóstico de Enfermedades Mentales de la Organización Mundial de la Salud, cuestión que fue ligeramente modificada en el 2001 cambiando la definición de desorden de identidad de género por el de trastorno de identidad de género (Vázquez, 2009).

Por su parte la Organización Mundial de la Salud (OMS) mantiene la transexualidad como un trastorno mental y de comportamiento asociado a cuestiones de identidad de género (Vázquez, 2009). Al clasificar la transexualidad como enfermedad mental, la OMS influye en el resto del mundo, exacerbando el estigma que ya existe sobre estas personas.

Los discursos biomédicos sobre transexualidad tienen el poder de hacer que las personas trans asuman el modelo biomédico que la define como patológica, tanto en el plano físico como mental, y que por tanto las transforma en personas diagnosticadas con un trastorno de identidad de género, sometiéndolas al sistema de salud imperante en cada país. Autores como Arán, Zaidhaft, Murta (2008), Lionço (Arán, Murta y Lionço, 2009), Gonçalves (2007), Butler (2006), Espín (2014), entre otros, critican la condición transexual como un trastorno mental.

Desde el derecho han primado dos posturas fundamentales sobre los estudios de la transexualidad: la de los autores que defienden que el derecho debe abstenerse de regular las cuestiones biológicas y la de los que consideran que debe ir más allá de las cuestiones biológicas, siguiendo las tradiciones sociales y culturales (Itxaso, 1999).

El presente trabajo ofrece una aproximación a la protección jurídica de las personas trans, en particular en el contexto de la Unión Europea y en el de América Latina. Para ello se analizan las soluciones jurídicas dadas en ambos contextos ante la ausencia de normas, así como los retos y perspectivas de la legislación para las personas trans. Con este objetivo fueron empleados diversos métodos de las ciencias jurídicas y sociales, como son el análisis histórico, para constatar la evolución jurídica doctrinal del tema; el método teórico-jurídico, para profundizar en conceptos y definiciones sobre el tema; el jurídico comparado, para verificar la correlación entre los modelos europeo y latinoamericano que protegen a las personas trans, y el hermenéutico analítico para analizar las diferentes posturas y soluciones jurídicas que se presentan tanto en el contexto europeo como en el latinoamericano.

\section{Desarrollo}

\section{Transexualidad en el derecho de la Unión Europea}

Actualmente se desconoce el número exacto de transexuales que viven en Europa. Existen estimativas que hablan de unos 30000 transexuales en el continente europeo (UE, 2016). 
La mayoría de los Estados miembros de la Unión Europea establecen que para obtener el reconocimiento legal es necesario haber realizado algún procedimiento, como cirugía, diagnóstico mental o tratamiento hormonal.

Desde la perspectiva jurídica, la Unión Europea no posee un consenso sobre algunos términos vinculados al aspecto legal de los transexuales, como son la discriminación y la identidad de género, lo cual lleva a que unos países traten el tema como discriminación sexual y otros como discriminación por orientación sexual. Esto trajo consigo que el Tribunal de Justicia de la Unión Europea (TJUE) entendiera que el asunto debía ser tratado como discriminación por razón de sexo, tal como se aprecia en el caso Sarah Margaret Richards v. Secretary of State for Work and Pensions (Sarah Margaret Richards v Secretary of State for Work and Pensions, 2006).

Esto implica que la distinción por razón de orientación sexual alcance también a los aspectos legales de la reasignación de sexo, pues en muchos casos la persona sufre discriminación debido al sexo al que se consideraba que pertenecía antes de la reasignación, según muestra el caso Sarah Margaret Richards v. Secretary of State for Work and Pensions, lo que contradecía el principio de igualdad entre hombres y mujeres respecto a la Directiva n. ${ }^{\circ}$ 79/7/CEE del Consejo, de 19 de Diciembre de 1978, relativa a realización progresiva del principio de igualdad entre hombres y mujeres.

En 2006, la Unión Europea adoptó la denominada "Directiva refundida" (2006/54/CE) buscando consolidar las disposiciones existentes sobre la aplicación del principio de igualdad de trato entre mujeres y hombres. La referida Directiva tiene por objeto garantizar la aplicación del principio de igualdad de oportunidades y de trato en materia de empleo y ocupación. Desde su preámbulo se observa una realidad jurídica sin precedentes, ya que en su Considerando Tercero se introduce una referencia explícita a la discriminación basada en la "reasignación de género", demostrando que el legislador de la Unión Europea tomó conciencia de la jurisprudencia del TJUE sobre la identidad de género. Analizando la Directiva se aprecia que existe una posición favorable a la protección jurídica de estas personas; tal es el caso del artículo n. ${ }^{\circ} 17$, que pide a los Estados miembros apoyar el combate a la discriminación con respaldo de la sociedad civil.

En resumen, el papel de la Corte Europea ha sido esencial en el abordaje del problema jurídico al que se enfrentan las personas trans, poniendo de manifiesto que la igualdad de estas personas se tiene que aplicar sobre la base del género adquirido después de una reasignación de género y no en relación con el sexo dado al nacer (P. v. S. and Cornwall County Council, 1994; K. B. v. National Health Service Pensions Agency, 2003; Sarah Margaret Richards v. Secretary of State for Work and Pensions, 2006).

Otra división sutil respecto a los países de la Unión Europea en el tema de las personas trans posibilita clasificar a los miembros en cuatro grupos: primero, aquellos que carecen de normas que aborden la materia; segundo, los que requieren tratamiento hormonal y/o cirugía para modificar el estatus legal; tercero, los que exigen la presentación de una prueba de disforia de género ante autoridad competente, y cuarto, los que permiten la autodeterminación del individuo.

1.1. Lituania. El primer ejemplo que podríamos colocar es el caso de Lituania que, aun cuando es un Estado miembro, no posee legislación referente al tratamiento a ser dado a las personas trans 
en su territorio. Este tema es resuelto a través de acuerdos y decisiones por la vía jurisprudencial. Esto evidencia que, aunque la Unión Europea intenta establecer un derecho uniforme hacia dentro del bloque, existen países que tiene posturas de resistencia en los temas de transexualidad y reconocimiento de sexo en sus ordenamientos internos.

1.2. Bélgica. Dentro del segundo grupo encontramos países como Bélgica, donde en 2007 era exigido como requisito el diagnóstico psiquiátrico obligatorio y el cumplimiento con un conjunto de medidas médicas incluyendo el tratamiento hormonal, una prueba de la "vida real" y las cirugías genitales de la reasignación envolviendo la esterilización. En esta legislación, el género jurídico solo podía modificarse en los casos en que existía una convicción irreversible de pertenecer al género "opuesto" y en los que se había realizado tratamiento médico.

En mayo de 2017, Bélgica emitió una nueva ley que establece un procedimiento administrativo para cambiar el sexo. Hasta esa fecha las personas trans solo podían cambiar su género legal y obtener documentos que reflejaran su identidad de género después de un largo procedimiento y una esterilización forzada.

Esta norma prevé el inicio del proceso ante el oficial en el registro civil, donde es presentada una declaración de que el sexo de su partida de nacimiento no corresponde con su verdadera identidad, a su vez el funcionario informa a la persona de las consecuencias jurídicas del proceso y cabe a esta decidir si continua o no. La declaración dada al agente se transfiere al procurador del Fiscal, dándose un plazo de 3 meses para emitir opinión. De ser negativa la respuesta, la persona inicia proceso ante un tribunal de familia. Pasado este tiempo se deben esperar seis meses para una segunda comparecencia ante el funcionario. Concluido el proceso y siendo favorable a la persona trans, el certificado de nacimiento se modifica, imitando el procedimiento danés.

La principal diferencia de esta ley respecto a otras existentes en la Unión Europea es que el procedimiento autoriza a los niños de tan solo 12 años a cambiar su apellido y su sexo cuando cumplen los 16 años. En este tipo de situaciones se requiere la asistencia de los padres junto con una declaración de un psiquiatra. La postura de Bélgica evidencia que, aun cuando las leyes van favoreciendo la inclusión de las personas trans, es necesario eliminar posturas arcaicas y discriminatorias como el caso de una declaración psiquiátrica que persiste.

1.3. Francia. Por su parte, Francia no ha tenido un proceso tranquilo respecto a otorgar derechos a las personas trans. En 1975 la Corte Suprema de Justicia decidió que los transexuales no podían cambiar su género legal basándose en el principio de que los individuos no pueden ejercer el control total de su personalidad jurídica (Caso B v. Francia, 1975). Este principio tenía por objeto garantizar el orden público, al tiempo que implicaba que la única posibilidad de cambiar ciertas características, como el nombre o el estado civil, eran los casos prescritos por la ley hasta la histórica sentencia en 1992 del Tribunal Europeo de Derechos Humanos (Caso B. v. France, 1992).

Como resultado de la sentencia del Tribunal Europeo de Derechos Humanos (Caso B. v. France, 1992), el Tribunal Supremo francés estableció que las personas transgénero podían obtener el reconocimiento legal si cumplían cuatro requisitos principales: ser diagnosticadas con "transexualismo"; someterse a tratamientos médicos y quirúrgicos; perder las características de 
su sexo biológico, y tener una apariencia y comportamiento social consistente con su identidad de género (Caso n. ${ }^{\circ}$ 91-11.900, 1992). Esto ha dado como resultado que algunos tribunales exijan a estas personas someterse a una evaluación de expertos, elevando el costo procesal para el solicitante.

En 2010, una Circular del Ministerio de Justicia francés aclaró los requisitos establecidos por el Tribunal Supremo en 1992 relativos a tratamientos médicos y quirúrgicos, indicando que no implicaban la necesidad de realizar cirugías de reasignación de género. Los tratamientos hormonales y otras cirugías se consideraron suficientes para certificar la irreversibilidad del proceso de transición.

Para poner fin a la práctica recurrente de exigir una evaluación de expertos, la Circular puso de relieve que la experiencia solo se requeriría en los casos en que surgiesen dudas en relación con la "transexualidad" del solicitante (UE, 2010). Sin embargo, en junio de 2012, la Corte de Casación confirmó la decisión del Tribunal de Apelación de París al negar el reconocimiento legal de género a una mujer transexual que se sometió a cirugía de reasignación genital en Tailandia y posteriormente se negó a someterse a una evaluación pericial (Caso n. ${ }^{\circ}$ 10-26.947, 2012). En este caso la Corte Suprema aclaró que el diagnóstico psiquiátrico y las pruebas del carácter irreversible de la transformación de la apariencia física eran necesarios para obtener el reconocimiento legal de género (Caso 11-14.515, 2013, y Caso 12-11.949, 2013).

En 2016, Francia modificó el Código Civil referente al procedimiento de actualización del registro de las personas trans. El artículo $n .^{\circ} 61-5$ de la ley otorga cambios legales tanto a la persona que ha alcanzado su mayoría de edad como a la emancipación de los menores. Por su parte el artículo n. ${ }^{\circ} 61$ establece que la falta de tratamiento médico, de cirugía o de esterilización no es un motivo para la denegación de una solicitud. Sin embargo, el artículo $n .^{\circ} 61-5$ obliga a la persona a probar suficientemente que su género al nacer no refleja el género adoptado. Este requisito está tan ampliamente redactado que es más que posible que los tribunales continúen solicitando al menos el tratamiento médico (Francia, 2017).

1.4. Reino Unido. Otro país europeo que también ha presentado ciertas polémicas en el asunto es el Reino Unido, donde la Ley de Reconocimiento de Género del Reino Unido de 2004 ha tenido varias altas y bajas, un ejemplo de ello es el caso Corbett v. Corbett (Corbett v. Corbet, 1970). Aquí la Corte del Reino Unido sostuvo que el sexo se fija al nacer, siendo inalterable y determinado por factores "objetivos" como los cromosomas, el fenotipo y los genitales.

La Ley del Reino Unido es muy detallada con respecto a la protección de la privacidad. Los únicos casos en que es apropiado el término "cambio de sexo" son aquellos en los que se comete un error de sexo al nacer y posteriormente se revela mediante una investigación médica adicional. La sentencia del tribunal posibilitó que fueran definidos los criterios para determinar el sexo de transexuales hasta la llegada de la Ley de Reconocimiento de Género de 2004. Esta norma tampoco logró resolver el conflicto jurídico de estas personas en el Reino Unido, pues determinó que el sexo de ellas se define de acuerdo con su certificado de nacimiento, lo que constituye una discriminación en razón del registro civil y no considera la autonomía de la voluntad de la persona trans al no identificarse con su sexo biológico. Al igual que en el caso francés es necesario realizar 
una cirugía de reasignación de sexo, un informe psicológico y ser mayor de edad. Un elemento a resaltar en el contexto inglés es la diferenciación entre los documentos de identidad de uso cotidiano y la negativa de modificar en el asiento registral del nacimiento el género de la persona trans. Este entendimiento evidencia que aún persisten posturas discriminatorias dentro de la Unión Europea al respecto del tema.

Hasta ahora el Tribunal Europeo ha tenido que ir contornando la situación de vulnerabilidad jurídica de las personas trans, pero sin dudas el caso Goowin v. Reino Unido de 2002 marca una vuelta de página en la temática, pues consideró que el Reino Unido había extrapolado sus límites al impedir que la Sra. Cristine Goowin (Caso Goowin v. Reino Unido, 2002), un transexual del sexo femenino convertido al sexo masculino, contrajera matrimonio con un hombre. En las alegaciones de la corte se aprecia la importancia de no tener exclusividad hacia la concepción del sexo biológico cromosómico sino también al aspecto psíquico y sobre todo respetar el sexo con el cual la persona se identifica.

1.5. Dinamarca. Ya la vanguardia de la Unión Europa está, sin dudas, en manos de Dinamarca. Aquí la legislación permite que las personas transexuales que han alcanzado su mayoría de edad (18 años) puedan determinar libremente su género legal. Aquí los derechos LGBT son algunos de los más extensos del mundo y de alta prioridad para el gobierno. Fue el primer país en el mundo que reconoció las uniones de personas del mismo sexo en forma de asociación registrada en 1989. Y en 2004 prohibió la discriminación por motivos de orientación sexual.

En 2014 Dinamarca aprobó una norma que determina el procedimiento legal de otorgar y reconocer los derechos de las personas trans. Una vez realizada la solicitud la persona tiene un período de seis meses para retractarse, pasado ese tiempo es otorgada una nueva identidad gozando de sus derechos como ciudadano. Esto evidencia que la presencia de posturas médicas como fundamentos de derechos acentúa la discriminación que sufren estas personas y más que justificaciones muchas veces constituyen impedimentos jurídicos para la manifestación de la voluntad de querer pertenecer a otro sexo de una persona (Dinamarca, 2014).

El matrimonio entre personas del mismo sexo es legal desde junio de 2012. Desde el año 2017, el transgénero ya no se considera un enfermo mental en Dinamarca. De hecho, el Gobierno danés consideró que la propuesta de desclasificar la orientación sexual en la Clasificación Estadística Internacional de Enfermedades y Problemas Sanitarios Conexos (CIE), presentada por primera vez en enero de 2014 y aún no revisada, tardaba demasiado en introducirse, y decidió actuar solo eliminándola de su sistema legal interno (Dinamarca, 2014).

1.6. Debate en el contexto europeo. Corte Europea de Derechos Humanos. En el contexto de la Unión Europea podemos acotar que las garantías de los derechos de las personas trans aún son susceptibles de perfeccionamiento y armonización. En un primer momento se aprecia que la interpretación de los artículos N. 8-12 del CEDH posee básicamente dos salidas: una marcada por el derecho que tiene la persona trans con respecto a su vida privada e intimidad a través de la concesión de documentos personales acordes a su nueva sexualidad, y otra marcada por el derecho que posee la persona trans a contraer matrimonio con otra persona de su mismo sexo cromosómico, pero que no sea el aparente bajo las reglas comunes. 
Si bien la CDEU no logró establecer soluciones definitivas a los problemas de las personas trans en el contexto europeo, ella constituye una referencia jurisprudencial para la región y al mismo tiempo demuestra la existencia de dificultades jurídicas sobre el tema. Varias son las interrogantes a resolver. ¿Cuál es la posición que debe adoptar un determinado país si una persona trans después de asumir su nueva identidad decide volver a su sexo cromosómico? ¿Cuál sería el tratamiento jurídico si el trans decide contraer matrimonio con una persona del sexo opuesto cromosómicamente, pero con el mismo sexo de su apariencia física? ¿Sería factible dar el mismo tratamiento jurídico a la persona que se sometió a una cirugía de reasignación de sexo y a aquella que no? ¿Cuál será el tratamiento jurídico dado en relación con hijos nacidos antes de su cambio de sexo biológico respecto a la guarda y cuidado? Ante estas interrogantes compete a la jurisprudencia y al legislador poseer un entendimiento equilibrado en favor de garantizar la protección de personas que se encuentran en grado elevado de vulnerabilidad.

\section{Transexualidad en el derecho latinoamericano}

El panorama latinoamericano no difiere mucho de la realidad existente en la Unión Europea. Las legislaciones latinoamericanas también poseen la misma clasificación: países que no poseen normas al respecto de los derechos de los transexuales, países que exigen tratamientos como cirugías o diagnósticos de disforia de género y los que abogan por la autodeterminación, además de aquellos que de cierta forma criminalizan las conductas homosexuales.

Además de la disparidad normativa en la región, tampoco existe un consenso respecto a las definiciones terminológicas utilizadas con relación a los derechos de las personas trans. Conceptos como delitos de odio, orientación sexual, discriminación, identidad de género y discurso de odio no poseen homogeneidad legislativa; muchas veces son empleados por el legislador como sinónimos cuando en realidad no lo son.

En el marco de la Organización de los Estados Americanos en 2008 fue adoptada la primera Resolución dedicada a la orientación sexual y de identidad de género, manifestando la preocupación de los Estados miembros por actos de violencia y violaciones de derechos humanos relacionados con cuestiones de identidad de género. En el año 2011 la OEA, a través de la AG/ RES 2653(XLI-O/11), solicita a la Comisión Interamericana de Derechos Humanos la realización de un estudio "sobre las implicaciones jurídicas y los desarrollos conceptuales y terminológicos relativos a orientación sexual, identidad de género y expresión de género", a fin de establecer una definición común dentro de la región que facilite la interpretación de términos que están ausentes en la mayoría de las legislaciones de los países.

La Convención Interamericana contra toda forma de discriminación e intolerancia define desde sus primeros artículos que constituye discriminación cualquier forma de exclusión restricción o preferencia en el ámbito público o privado (OEA, Convención Interamericana contra toda forma de discriminación e intolerancia (A-69), 6 de mayo de 2013). También establece en su artículo n. ${ }^{\circ} 1$, párrafo segundo, que la discriminación puede estar basada en motivos de orientación sexual, identidad y expresión de género. Esta Convención constituye un referente obligado dentro de América Latina para tratar cuestiones de identidad de género. 
De manera general en Latinoamérica solo dos países ofrecen garantías constitucionales a los derechos entre homosexuales, tal es el caso de las Constituciones de Bolivia (Bolivia, Constitución de Bolivia, 2009) y Ecuador (Ecuador, 2008). Ambas Cartas Magnas prohíben de manera expresa actos discriminatorios basados en la identidad de género. El resto de los países, de modo general, asume posiciones como la de Brasil (Brasil, Constitución de la República Federativa de Brasil, 1988) o la de Cuba (Cuba, Constitución de la República de Cuba, 2019), protegiéndolos dentro de los principios fundamentales, o mediante la interpretación de estos por parte de la jurisprudencia.

2.1. Caribe. La región del Caribe presenta la situación más crítica en cuestiones de identidad de género, ya que en muchos de los países de esta región se establecen como crímenes diferentes manifestaciones de esta identidad. El Informe de la OEA sobre Violencia contra las personas LGBT de 2015 destaca los países que criminalizan las relaciones sexuales entre personas del mismo sexo y las identidades de género no normativas. Un total de 11 países (Antigua y Barbuda, Barbados, Belice, Dominica, Granada, Guyana, Jamaica, Santa Lucía, San Vicente y las Granadinas, San Cristóbal y Nieves, Trinidad y Tobago) criminalizan de una forma u otra la sodomía (OEA, 2015).

2.2. Cuba. En el caso de Cuba podemos establecer una equiparación con la realidad de Lituania, donde no existe norma jurídica que establezca los derechos de las personas trans de forma clara. Cuba posee una ausencia total de legislación en relación con la identidad de género, lo que la coloca en la lista de países que no reconocen ni equiparan las uniones legales entre homoafectivos, transgéneros, bisexuales, homosexuales. El Código de Familia (Ley n. ${ }^{\circ}$ 1289/1975), en su artículo $n .^{\circ} 2$, es bien claro al reconocer de manera exclusiva el matrimonio entre hombre y mujer (Cuba, 1975, frebrero 14). Esto imposibilita que las personas con identidad de género diferente al modelo patriarcal puedan tener un disfrute pleno de su sexualidad, incentivando prácticas discriminatorias, lo que evidencia la ausencia de derechos y deberes en las relaciones homoafectivas en la isla.

La Constitución vigente de 2019 destaca someramente algunos elementos que de manera análoga permiten realizar una interpretación de los derechos inherentes a la personalidad apoyándose en el artículo $n .^{\circ} 40$, que garantiza la inviolabilidad de la persona humana; en el artículo $\mathrm{n} .^{\circ} 41$ sobre el respeto a los derechos humanos, y en el artículo $n .^{\circ} 42$, en el que se dispone el combate a la discriminación de cualquier conducta lesiva contra la dignidad de la persona humana (Cuba, 2019, febrero 24).

Si bien estos artículos son utilizados ante la ausencia de referentes legislativos, resultan insuficientes a la hora de minimizar las cuestiones de discriminación a que son sometidas estas personas. Como norma general es un tanto estereotipada, y refuerza los patrones físico-biológicos de la sociedad patriarcal, segmentando la identidad de género de aquellos individuos que no se ajustan a esta clasificación.

Por otro lado, la Ley n. 59 de 8 de noviembre de 1988 del Código Civil Cubano (Cuba, 1987, julio 16) establece en su Sección Cuarta, "Derechos inherentes a la personalidad", artículo n. ${ }^{\circ} 38$, que los derechos inherentes a la personalidad están consagrados en la Constitución, lo que deja a los operadores del derecho sin muchas salidas para casos de identidad de género, pues remite a una norma que ya es omisa de por sí. 
La Ley n. ${ }^{\circ} 51$ de 15 de julio de 1985 del Registro del Estado Civil de Cuba, dispone en su artículo n. ${ }^{\circ} 73$ que el sexo en el asiento registral al nacer es definido por el sexo biológico (masculino/ femenino), cuestión que se revierte con la fijación de la identidad de género del individuo transexual. Además, se dispone que los asientos de las oficinas registrales solo podrán ser modificados $o$ anulados mediante sentencia judicial del tribunal competente, negando la posibilidad de resolver cuestiones de este tipo por la vía administrativa.

No fue sino hasta el 2013 que la legislación cubana tuvo una norma que abordase las cuestiones de discriminación por motivo de identidad de género. El Código del Trabajo, que saliera a la luz el 16 de junio de 2014, estableció como principio obligatorio la no discriminación por motivos de identidad de género, sentando el primer precedente normativo que aborda el asunto de manera expresa (Cuba, 2014, junio 17).

Tampoco las legislaciones actuales abogan por eliminar la discriminación existente a estas personas, tal es el caso de la nueva Constitución. El Capítulo III, "Las Familias", establece en su artículo 81 que toda persona tiene derecho a fundar una familia, cualquiera que sea su forma, alegando que la protección jurídica de los diferentes tipos de familia es regulada en ley (Cuba, 2019 , febrero 24). En este caso, de ser aprobado el texto constitucional, colocaría a los operadores del derecho en una disyuntiva ante una norma en blanco $u$ omisa, pues no existe un cuerpo normativo que regule las relaciones familiares para personas del mismo sexo o personas trans. Esto implicaría una mudanza estructural profunda que a largo plazo pueda garantizar de manera efectiva tanto los compromisos con los tratados internacionales de los cuales Cuba es parte como la concesión de derechos y deberes de sus nacionales con preferencias sexuales que salen del canon patriarcal y heterosexual.

Vale destacar que el sistema jurídico cubano no admite como fuente formal de derecho la jurisprudencia, lo que dificulta aún más la situación de las personas trans y de los conflictos jurídicos cuyo basamento es la identidad de género. Es una práctica consolidada entre los operadores del derecho no enunciar directamente violaciones de tipo constitucional con fundamentos de derecho ante lagunas legislativas, razón que enfatiza la necesidad de un tribunal constitucional que garantice estos derechos.

En la jurisprudencia cubana al respecto se destacan tres casos hasta el presente: el primero, en el año 1998, demanda presentada en la Sala Segunda de lo Civil y Administrativo del Tribunal Popular de la Ciudad de la Habana, radicada en el Expediente n. ${ }^{\circ}$ 128/1996 de 14 de enero de 1998; el segundo, ante el Tribunal Municipal Popular del San Miguel del Padrón en Ciudad de La Habana, Expediente n. ${ }^{\circ}$ 31/2001 de fecha 28 de febrero de 2002; y el tercero, ante el Tribunal Municipal de Artemisa de la Ciudad de La Habana, expediente N. ${ }^{\circ} 285$ de fecha 30 de septiembre de 2003.

En todos los casos fue realizada la cirugía de reasignación de sexo fuera de Cuba, lo que permitió aportar pruebas documentales, testificales, periciales, así como la confesión judicial. Todos los demandantes declararon sentir inconformidad entre el sexo biológico y físico, lo que llevó a adoptar un procedimiento quirúrgico para establecer un equilibrio entre el aspecto físico y emocional, rebasando el estrecho marco de la homosexualidad. Ante la ausencia de norma 
las decisiones fueron sustentadas bajo los artículos constitucionales n. ${ }^{\circ} 9, n .{ }^{\circ} 41$ y n. ${ }^{\circ} 54$ de la derogada Constitución de 1975.

Si bien las sentencias proponen un camino para estas situaciones que afrontan los transexuales, ellas no ofrecen una solución definitiva, ya que en el caso de Cuba la jurisprudencia no constituye fuente formal de derecho como en países de Europa, lo que provoca que cada caso sea interpretado de una manera diferente.

Ambas sentencias no logran resolver el fondo del asunto de manera definitiva, pues a pesar de que el fallo fue favorable a los demandados, solo determinó la corrección en nota marginal del certificado de nacimiento en cuestiones relativas al nombre y al sexo, amparados en el procedimiento quirúrgico realizado en el exterior.

Actualmente es posible modificar la foto y el nombre en el documento de identidad, sin que exista un fundamento legal para ello. Al cierre de 2018, según datos divulgados por especialistas del Centro Nacional de Educación Sexual de Cuba, habían sido realizadas un total de 30 cirugías de reasignación de sexo, 28 hacia el sexo femenino y 2 hacia el masculino. Hasta el momento solo es posible iniciar proceso judicial para modificar el asiento registral (sexo femenino/masculino) si no es realizada cirugía de reasignación de sexo tanto fuera como dentro de Cuba, quedando sin solución jurídica aquellas personas transexuales que no apuestan por una cirugía y que sin dudas representan un universo mayor.

Esta realidad jurídica ubica a Cuba en el medio de una clasificación que hemos venido abordando. Por un lado, se apunta como la Lituania latinoamericana al carecer de legislación al respecto y por otro como la Francia caribeña al exigir la cirugía como elemento esencial para la modificación registral de la persona trans. Esto evidencia que no hay un entendimiento jurídico uniforme por parte de los operadores del derecho en Cuba.

2.3. Brasil. La realidad jurídica de Brasil en este ámbito no difiere mucho de la cubana o la francesa, existiendo pocos cuerpos normativos que regulen los derechos de las personas trans, lo cual provoca la necesidad de interpretaciones jurisprudenciales que no siempre son uniformes en el territorio nacional. Desde 1999 el Consejo Nacional de Psicología en Brasil determinó que el psicólogo deberá contribuir con su conocimiento a eliminar prejuicios, discriminación, estigmatización contra aquellas personas que presentan prácticas homoeróticas, dejando de ser tratadas como trastornos mentales las cuestiones de identidad de género (Brasil, 1999, marzo 22). Solo después de 10 años el Consejo Federal de Medicina autorizó a través de la Resolución CFM n¹.955/2010, la cirugía de transgenitalización como tratamiento secundario al caso del transexualismo. El propio cuerpo legal dispone de criterios mínimos para la ejecución de la cirugía, tal como refiere el artículo 3, destacándose la inconformidad con el sexo anatómico natural, deseo expreso de eliminar los genitales y perder las características propias del sexo al ganar las del sexo opuesto; permanencia de ese disturbio de manera continua por lo mínimo de 2 años y ausencia de otros trastornos mentales (Brasil, 2010, septiembre 3). Además, para efectuar la cirugía se exige un acompañamiento multidisciplinar por más de 2 años de psicólogos, endocrinólogos, psiquiatras, cirujanos y un asistente social, quienes evalúan la existencia de un diagnóstico de transexualismo en personas mayores de 21 años y las características físicas del individuo. 
Ante la existencia de estas normativas y en la ausencia de legislación que solucionase las cuestiones de identidad de género, en el 2011 el Supremo Tribunal Federal (STF) se pronunció a favor de la unión homoafectiva a través de una Acción Directa de Inconstitucionalidad, expresando la prohibición de discriminación de las personas en razón de sexo, sea en el plano de la dicotomía (hombre/mujer), o en el plano de orientación sexual (Brasil. STF, 2011). Esta decisión no fue de estricto cumplimiento por parte de los tribunales en Brasil, lo que llevó a diversas interpretaciones sobre el asunto por parte de los magistrados. En consonancia con esta realidad el Consejo Nacional de Justicia emitió la Resolución n. 175 de 14 de mayo de 2013, estableciendo que tanto las uniones estables como los casamientos entre personas del mismo sexo sean equiparadas a las relaciones jurídicas de esta naturaleza que la ley concede a las personas heterosexuales (Brasil, 2013, mayo 14).

Esta normativa trae ciertas garantías jurídicas ausentes hasta el momento para las personas trans, lo que evidencia la voluntad política del Estado brasileño de alcanzar los escaños más altos de protección en la región. Es importante resaltar que la jurisprudencia brasileña no posee un entendimiento uniforme acerca de la aplicación de las normativas vigentes y de cómo ellas deben ser interpretadas en los diferentes casos que llegan a los tribunales.

Un ejemplo que ilustra lo anterior es el Recurso Extraordinario n. $670422 \mathrm{RG} / \mathrm{RS}$ contra la decisión de la 8va Cámara Civil del Tribunal de Río Grande del Sur sobre el cambio de sexo de una persona transexual. En este proceso fue concluida la imposibilidad de cambiar el sexo en los documentos registrales sin la realización de la cirugía de reasignación sexual. Para ello el foro entendió que debía ser colocada la palabra transexual en lugar de sexo, concluyendo que resultaba imposible la modificación de género. Ante esta decisión, el Superior Tribunal de Justicia (STJ) decidió que se trataba de un hecho que evidenciaba una violación constitucional en cuanto al principio de la dignidad de la persona humana y que competía al Supremo Tribunal Federal (STF) realizar un pronunciamiento por el impacto social de la decisión, a lo que este este respondió reafirmando la inconstitucionalidad de la decisión.

A primera vista podría parecer que Brasil encabeza la segunda vanguardia en el tema en la región, pero nada más alejado de la realidad. La carencia de una interpretación uniforme en el tema por parte de la jurisprudencia nacional y la ausencia de normas claras que protejan los derechos de estas personas de manera más directa, demuestran abiertamente las situaciones de discriminación a que son sometidas estas personas. En 2017 una nueva decisión del STJ autoriza la alteración del registro civil sin realizar cirugía. En septiembre la justicia del Distrito Federal (DF), una instancia inferior, a manos del juez Waldemar Claudio de Carvalho, determina a través de una decisión cautelar que los psicólogos puedan tratar gays y lesbianas como enfermos mentales y hacer terapias de reversión sexual. Con esta acción se busca suspender la Resolución n. ${ }^{\circ}$ 01/1999 del Consejo de Medicina y retroceder en el equilibrio logrado hasta entonces en este asunto.

Vale cuestionarse en qué nivel se encuentra le legislación brasileña en temas de identidad de género. Podríamos pensar que el panorama jurídico está próximo a promulgar una normativa al respecto, pero la situación jurídica es compleja y alarmante, pues los operadores del derecho no tienen una interpretación uniforme de los casos que pasan por sus manos y la falta de legislación propicia la ocurrencia de hechos como el caso del DF. Esta dicotomía entre las decisiones de 
instancias superiores e inferiores está dada por la promulgación de jurisprudencia no vinculante y por el hecho de que pocas veces son enunciadas como referentes para emitir decisiones sobre el tema.

2.4. Bolivia. El caso boliviano se puede equiparar a la situación belga, donde la legislación del país evolucionó hacia la eliminación de exigencias de pruebas documentales para otorgar los cambios registrales.

En el año 2006 en Bolivia solo era posible el cambio de sexo en los documentos a través de un proceso judicial, donde era necesario aportar pruebas documentales que podían consistir en un certificado médico, una pericia psiquiátrica o fotografías que demostraran la apariencia actual y anterior de la persona. Para esta comunidad LGBT, es denigrante tener que aportar pruebas que comprueben su identidad sexual cuando esto constituye un principio consagrado en la Constitución de Bolivia (Bolivia, 2009, febrero 7). Diez años después el país se ubica a la cabeza junto con Argentina en la legislación de punta y determina que se realice el cambio de sexo. La nueva normativa, Ley n. ${ }^{\circ} 807$ de 21 mayo de 2016, dispone los requisitos y el procedimiento para cambio de sexo de las personas trans en los documentos registrales (Bolivia, 2009, febrero 7; Bolivia, 2016, mayo 21). Entre las principales exigencias están las siguientes: carta de solicitud, certificado de nacimiento original, certificado de descendencia, certificado del estado civil y certificado de antecedentes penales. El cambio permite a la persona el goce y disfrute de todos los derechos fundamentales, quedando prohibido el uso de cualquier documento anterior una vez asumida la nueva identidad.

Esto demuestra que, aunque no existe uniformidad en la región latinoamericana, varios países buscan avanzar hacia la concesión de los derechos de las personas trans, evidenciándose un progreso jurídico favorable a la eliminación de estigmas y discriminaciones que han prevalecido por décadas.

2.5. Argentina. Ya la realidad argentina es más consonante con la vanguardia dinamarquesa otorgando varios derechos y facilidades a las personas trans en cuestiones basadas en la autodeterminación del individuo.

En el año 2010 Argentina autoriza el matrimonio entre personas del mismo sexo, contemplando idénticos derechos y garantías legales que los reconocidos a parejas heterosexuales, e incluyendo derechos de adopción y beneficios jubilatorios. En el 2012 fue promulgada la emblemática Ley de Identidad de Género n. ${ }^{\circ} 26.743$ de 9 de mayo de 2012, en la cual se define que la identidad de género es la vivencia interna e individual del género tal como cada persona la siente, pudiendo corresponder o no con el sexo asignado al momento de nacer. Entre los principales destaques de esta norma se encuentra la posibilidad de proceder a la rectificación registral del sexo sin que sea obligatorio realizar la reasignación genital total o parcial, ni el tratamiento hormonal o psicológico, cuestión que para la época constituía toda una novedad jurídica en materia de identidad de género (Argentina, 2012, mayo 9). Esta ley fue de las primeras en combatir la patologización de las personas trans y constituye un referente en América Latina para el tratamiento jurídico de los derechos de estas personas. 
Los primeros años fueron bien protagónicos en el cambio de la identidad de las personas trans, realizando entre 1854 y 1336 solicitudes. Entre tanto de 2014 a 2016 fueron realizadas 763, 804 y 749 (Argentina, 2017). La mera solicitud ante el Registro Nacional de personas efectuadas por mayores de 18 años es más que suficiente para proceder a la modificación de los documentos, lo que resulta similar a países de la Unión Europea como Dinamarca o Irlanda que siguen las posturas más vanguardistas en esa región.

A nueve años de la entrada en vigor de la Ley Transgénero en Argentina aún este país se encuentra con situaciones de discriminación en el ámbito educacional, laboral y social. La discriminación de estas personas no acaba con la promulgación de una regulación específica para ellos. La sociedad necesita abandonar las posturas patriarcales que durante siglos dominaron en los diferentes sistemas jurídicos. La ausencia de una legislación trans en materia laboral, si bien ha limitado una mayor integración social de estas personas, no ha sido un freno a la voluntad estatal, pues en febrero de 2017 fueron integradas tres mujeres trans al trabajo de la Secretaría Legislativa (Marina, 1 de febrero de 2017).

2.6. Debate en el contexto latinoamericano. El sistema interamericano de derechos humanos no es exactamente un modelo igual a la Corte de Justicia de la Unión Europea a la que nos referimos en la primera parte de este artículo. Ante la Corte Interamericana son presentados casos en los que se alega violación a los derechos protegidos en la Convención de Derechos Humanos de 18 de julio de 1978 y en los cuales haya sido agotada toda la vía procesal en el ámbito interno. Entre las principales críticas a la corte están la exclusividad de los Estados parte para presentar casos, la politización de las decisiones, así como la terminación del proceso con sentencias inapelables, obligatorias y definitivas.

Varias son las violaciones a los derechos humanos a que se enfrentan las personas trans en el contexto latinoamericano y pocas de ellas llegan al ámbito de la OEA. Un caso interesante es el caso penal: Caso 12.982 Luis Alberto Rojas Marín v. Perú (15 de abril 2009), donde se dictaminó como abuso la violación que sufrió una detenida por un agente del Estado durante la reclusión; este es un hecho censurable teniendo en cuenta la vulnerabilidad de la víctima ante el abuso cometido por los agentes. Varios son los artículos de la Convención América sobre Discriminación que deben ser cumplidos por el referido país ante este caso, como son los artículos $\mathrm{n}{ }^{\circ} 5,7$ y11, entre otros (OEA, 6 de mayo de 2013).

Se destaca que varias definiciones utilizadas se apoyan en decisiones de la Corte Europea ante casos similares, lo que evidencia el importante papel que juegan estas decisiones en la jurisprudencia internacional, siendo referentes para resolver conflictos o violaciones de derecho que ultrapasan el marco regional y que buscan el respeto de la dignidad de la persona humana por encima de todo. Esto refuerza el predominio del modelo eurocéntrico en la jurisprudencia internacional, al mismo tiempo que refuerza el papel periférico del derecho latinoamericano en el contexto global.

Otro caso interesante en el marco latinoamericano es el Caso Karen Atala e hijas, Caso 12.502 (17 de septiembre de 2010), donde se pide que se responsabilice al Estado de Chile por la violación del derecho de igualdad y no discriminación que se establece en el artículo n. ${ }^{\circ} 24$ 
de la Convención Americana. El caso fue presentado desde 2008 y solo en 2010 llegó a feliz solución. La dominación de la heteronormatividad (Rey, 2014) que impera en la región agudiza las situaciones de discriminación de las personas trans y conduce a situaciones de violencia que muchas veces terminan con la vida de estas personas.

Lopes (2016), cuando aborda el estudio del caso, destaca que la Corte Interamericana de Derechos Humanos (Corte IDH) fue más osada que la Corte Europea, pues entendió que la prohibición de la discriminación por su orientación sexual equivale a una exigencia de tratamiento jurídico idéntico entre personas homosexuales y heterosexuales. Si bien no es un caso donde se decide sobre la persona transexual, el enfoque que se combate apunta en la misma dirección: el combate contra estereotipos sociales y jurídicos que defienden la existencia de legislaciones basadas en modelos patriarcales.

\section{Conclusiones}

La permanencia de la transexualidad como un trastorno de identidad de género en el ámbito de la Organización Mundial de la Salud constituye un impedimento para que países como los de la región del Caribe avancen hacia la despenalización y la no criminalización de la transexualidad. Resulta necesaria la existencia de tratados internacionales que contribuyan a minimizar las situaciones de violencia, vulnerabilidad y discriminación que sufren estas personas.

Ciertamente, la Unión Europea constituye un referente tanto desde el punto de vista legislativo como jurisprudencial para resolver las cuestiones de ausencias normativas de las personas transexuales, pero exportar el modelo europeo hacia la realidad latinoamericana no resulta la solución más prudente debido a las diferencias jurídicas que separan ambas regiones.

La realidad latinoamericana es un tanto diferente a la de la Unión Europea, porque aún persisten países que condenan y criminalizan a las personas transexuales. Otro elemento en destaque es la ausencia de tratados regionales que concedan mayores garantías a estas personas, siendo una legislación fragmentada y generalizada, dado que son protegidos los derechos a través de cláusulas generales del Derecho Constitucional cuando se carece de legislación, lo que no garantiza una verdadera protección.

Ambas regiones poseen una disparidad legislativa cuando se mira por dentro de los países miembros, esto es provocado por la ausencia de tratados internacionales que constituyan una guía para el tratamiento legislativo del tema. Esta situación también obliga a la jurisprudencia a ocupar un lugar protagónico en la concesión de derechos y garantías fundamentales de estas personas, supliendo las lagunas legislativas que persisten en el tema y que están lejos de desaparecer.

Los trans necesitan hoy de un amparo jurídico universal más allá de fronteras bloques o regiones, como el único camino para ir resolviendo su estatus jurídico ante la sociedad mundial. El papel de los tribunales debe irse reduciendo en favor del surgimiento de legislaciones que garanticen mejores derechos a estas personas. La interpretación del derecho y la analogía no constituyen la solución definitiva a la realidad de estas personas. 


\section{Referencias bibliográficas}

Arán, M., Zaidhaft, S. y Murta, D. (2008). Transexualidade: corpo, subjetividade e saúde coletiva. Psicologia \& Sociedade, 20(1), 70-79. Recuperado de http://www.scielo.br/scielo.php?pid=S0102-71822008000100008\&script=sci_abstract\&tlng=pt

Arán, M., Murta, D. y Lionço, T. (2009). Transexualidade e saúde pública no Brasil. Ciência \& Saúde Coletiva, 14(4), 1141-1149. Recuperado de http://www.scielo.br/scielo.php?pid=S1413-81232009000400020\&script=sci_abstract\&tlng=pt

Argentina (2012, mayo 9). Ley 26.743/2012. Identidad de Género. Buenos Aires, Argentina: Boletín Oficial N. ${ }^{\circ} 4$.

Argentina (2017). Nuevas realidades, nuevas demandas. Desafíos para la medición de la identidad de género en el censo de población de Argentina. Buenos Aires, Argentina: Ministerio de Hacienda.

Benjamin, H. (1966). The transsexual phenomenon. Recuperado de http://www.mut23.de/texte/Harry\%20Benjamin\%20-\%20The\%20Transsexual\%20Phenomenon.pdf

Bolivia (2009, febrero 7). Constitución de Bolivia. Gaceta Oficial N. 107. La Paz, Bolivia: Gaceta Oficial.

Bolivia (2016, mayo 21). Ley N. 807/2016. Ley de identidad de género. Gaceta Oficial, núm.4.

Brasil (1988, octubre 5). Constitución de la República Federativa de Brasil. Brasilia, DF, Brasil: Diario Oficial de la Unión, núm.130.

Brasil (1999, marzo 22). Resolución 001/1999. Normas de Actuación de los Psicólogos en relación con la cuestión de orientación sexual. Brasilia, D. F., Brasil: Diario Oficial de la Unión.

Brasil (2010, septiembre 3). Resolución CFM N. ${ }^{\circ}$ 1.955/2010. Dispone la cirugía de transgenitalismo. Brasilia, D. F., Brasil: Diario Oficial de la Unión, núm. 2.

Brasil (2013, mayo 14). Resolución 175/2013. Dispone la habilitación para celebrar casamientos civiles y uniones estables entre personas del mismo sexo. Brasilia, D. F., Brasil: Diario Oficial de la Unión.

Brasil. STF (2011). Acción Directa de Inconstitucionalidad. ADPF 132/2011 RJ - Río de Janeiro (Tribunal Supremo Federal, Plenario, mayo 5, 2011).

Butler, J. (2006). Precarious Life: The Powers of Mourning and Violence. Londres-New York: Verso.

Caso 11-14.515 (Francia. Corte Suprema de Casación, Sección Civil 1ª , febrero 13, 2013).

Caso 12-11.949 (Francia. Corte Suprema de Casación, Sección Civil 1ª , febrero 13, 2013). 
Caso B. v. Francia, 73-10.615/1975 (Francia. Corte Suprema de Casación, Sección Civil 1ª , diciembre 16, 1975).

Caso B. v. France, Caso N. ${ }^{\circ} 13343 / 87$ (Tribunal Europeo de Derechos Humanos, marzo 25, 1992).

Caso Goowin v. Reino Unido (Tribunal Europeo de Derechos Humanos, julio 11, 2002).

Caso n. ${ }^{\circ}$ 10-26.947 (Francia. Corte Suprema de Casación (Sección Civil 1a) junio 7, 2012).

Caso n. ${ }^{0}$ 12-11.949 (Francia. Corte Suprema de Casación, Sección Civil 1ª , febrero 13, 2013).

Caso no. 91-11.900 (Francia. Corte Suprema de Casación, Sección Civil 1ª diciembre 11, 1992).

Cauldwell, D. O. (1949). Oddities of Sexual Behavior. Berlin: B-840.

Corbett v. Corbet (Reino Unido. Tribunal Supremo, mayo 14, 1970).

Cuba (1975, febrero 14). Código de Familia N. 1289/1975. Código de Familia ley N. 1289/1975. La Habana, Cuba: Gaceta Oficial.

Cuba (1987, julio 16). Código Civil de Cuba. Ley N. 59. La Habana, Cuba: Gaceta Oficial, N. 83.

Cuba (2014, junio 17). Ley N. ${ }^{\circ}$ 116. Código del Trabajo. La Habana, Cuba: Gaceta Oficial de la República de Cuba.

Cuba (2019, febrero 24). Constitución de la República de Cuba. La Habana, Cuba: Gaceta Oficial.

Dinamarca (2014, junio 12). Denmark becomes Europe's leading country on legal gender recognition. Recuperado de http://www.lgbt-ep.eu/press-releases/denmark-becomes-europes-le

Ecuador (2008, octubre 20). Constitución de Ecuador. Registro Oficial, núm. 165. Quito, Quito, Ecuador: Registro Oficial, núm. 165.

Espín, M. C. (2014). El programa nacional de educación sexual en la estrategia cubana de desarrollo. La Habana, Cuba: Abril.

Fisk, N. (1973). The How, What and Why of a Disease. New York, US.

Francia (2017, marzo 2). Código Civil Francés. París, Francia: Diario Oficial.

Gonçalves, À. A. (2007). Transexualidae: entre os discursos jurídicos e médico. Revista de Direito Uniceub, 17. Hirschfeld, M. (1923). The Homosexuality of Men And Women. New York, US. 
Itxaso, M. E. (1999). La Transexualidad. Jurisprudencia y Argumentación Jurídica. Granada: Biblioteca Comares.

K. B. v. National Health Service Pensions Agency, Caso C- 117/01 (Tribunal Europeo de Derechos Humanos, junio 10, 2003).

Marina, R. (1 de febrero de 2017). Cupo laboral trans: incorporan a tres transexuales a la Cámara de Diputados bonaerense. La Nación. Recuperado de https://www.lanacion.com.ar/sociedad/cupo-laboral-trans-incorporan-a-tres-transexuales-a-la-camara-de-diputados-bonaerense-nid1980908

Lopes, I. (2016). El reconocimiento transnacional del matrimonio entre personas del mismo sexo o de la pareja homosexual y los recientes desarrollos en Brasil y en el Mercosur. Anuario Español de Derecho Internacional Privado, (16), 759-792. Recuperado de https://iprolex.files.wordpress.com/2016/08/el-reconocimiento-transnacional-del-matrimonio-entre-personas-del-mismo-sexo-o-de-la-pareja-homosexual-y-los-recientes-desarrollos-en-brasil-y-en-el-mercosur.pdf

Money, J. (1997). Principles of developmental sexology. New York, NY, US: Continuum.

OEA (2015). Informe sobre Violencia contra personas LGBT. San José, Costa Rica: OEA. http://www.oas.org/.

OEA (6 de mayo de 2013). Convención Interamericana contra toda forma de discriminación e intolerancia (A69). La Antigua, Guatemala: OEA.

OEA (6 de mayo de 2013). Convención Interamericana contra toda forma de discriminación e intolerancia(A-69). La Antigua, Guatemala: OEA.

P v S and Cornwall County Council, Caso C-13/94 (Tribunal Europeo de Derechos Humanos, abril 30, 1994).

Rey, F. (2014). Caso de la Corte Interamericana de Derechos Humanos "Atala Riffo y niñas contra Chile" (24 de febrero de 2012). Cuestiones Constitucionales, (30), 237-249. Recuperado de http://www.redalyc. org:9081/articulo.oa?id=88531884010

Sarah Margaret Richards v Secretary of State for Work and Pensions, Caso C-423/04 (Tribunal Europeo de Derechos Humanos, abril 27, 2006).

Stoller, R. (1982). A experiência transexual. Rio de Janeiro, Brasil: Imago.

UE (2016). Ser trans en la UE. Análisis comparativo de los datos de la encuesta a las personas LGBT en la UE. Viena, Austria: Agencia de los Derechos Fundamentales de la UE.

UE, D. H. (2010, mayo 14). Demandas civiles por cuestiones de cambio de sexo. Circular núm. CIV/07/10. Bruselas, Bélgica: Comisión Europea de Derechos Humanos. 
Vázquez, F. (2009). Del sexo dicotómico al sexo cromático. La subjetividad transgenérica y los límites del constructivismo. Sexualidad, Salud y Sociedad, (1), 63-88. Recuperado de https://www.e-publicacoes.uerj.br/ index.php/SexualidadSaludySociedad/article/view/10/124

WPATH De-Psychopatholisation Statement of 26 May 2010. (s.d.). Consulté le 15 Septiember 2017, sur http:// www.wpath. org/uploaded_files/140/files/ de-psychopathologisation\% 205-26-10\%20on\%20letterhead. pdf>. 